\title{
Development of an intelligent surgical training system for Thoracentesis
}

\author{
Nakawala Hirenkumar*, Ferrigno Giancarlo, De Momi Elena \\ Department of Electronics, Information and Bioengineering (DEIB), Politecnico di Milano, Piazza Leonardo da Vinci, \\ Milan, 20133, Italy. \\ Email:\{hirenkumar.nakawala*, giancarlo.ferrigno, elena.demomi\}@polimi.it
}

\begin{abstract}
Surgical training improves patient care, helps to reduce the surgical risks, increases surgeon's confidence, and thus enhances overall patient safety. Current surgical training systems are more focused on developing technical skills, e.g. dexterity, of the surgeons while lacking the aspects of context-awareness and intra-operative real-time guidance. Thus, there is indeed a need to create an intelligent training system to raise context awareness in surgery. Context-aware intelligent training systems interpret the current surgical situation and help surgeons to train on surgical tasks. As a prototypical scenario, we chose Thoracentesis procedure in this work. We designed the context-aware software framework using the surgical process model encompassing ontology and production rules, based on the procedure descriptions obtained through textbooks and interviews, and on ontology-based and marker-based object recognition, where the system tracked and recognised surgical instruments and materials in surgeon's hands and recognised surgical instruments on the surgical stand. The ontology was validated using annotated surgical videos, where the system identified "Anaesthesia" and "Aspiration" phase with $100 \%$ relative frequency and "Penetration" phase with $65 \%$ relative frequency. The system tracked surgical swab and $50 \mathrm{~mL}$ syringe with approximately $88.23 \%$ and $100 \%$ accuracy in surgeon's hands and recognised surgical instruments with approximately $90 \%$ accuracy on the surgical stand. The user training with the system showed the similar results as the traditional mentor-based training regime. The developed surgical software framework could be used as a new tool for the context awareness and decision-making during the surgical training.
\end{abstract}

Keywords: Surgical training; Thoracentesis; ontology; production rules; tracking; object recognition

\section{Introduction}

Surgery is a highly complex process that requires excellent technical skills [1] e.g. dexterity, procedural knowledge, nontechnical skills, e.g. cognitive skills (context awareness, decision-making, and planning) [2] [3], and clinical skills [4] e.g. assessment, diagnosis to achieve better patient outcomes and to improve the performance quality in the operating theatre. Recently, following the emergence of new technologies, e.g. robot-assisted surgeries, the focus on surgical care is moving also towards the quality rather than only on the quantity of the procedure performed. The quality was also further enhanced with the recent advancements in surgical training regimes e.g. by employing surgical simulators [5] [6] in advanced surgeries, e.g. thoracic surgeries. In a particular study, researchers [7] have shown that technical errors account for only $4.3 \%$ during surgery, while most of the errors are related to non-technical errors especially clinical decision-making process. Although context awareness is an important cognitive skill of the surgery and part of the decision making, most of the current surgical training environments are focused on improving only surgeons' technical skills. Surgeon's capacity to make the intra-operative situational judgement is influenced by surgeon's technical capabilities, patient's conditions, and the competence of assisting trainee [8]. As traditional training methods are designed on mentoring by different expert surgeons, they could be highly variable. Thus, the competence of expert surgeons is also of prime importance with the current mentor-based training surgical regimes. Judgements related to contextual awareness are influenced by mental models of the surgeon's experience stored in the memories of intervention and clinical situations [8]. Novice surgeons who learn performing high-risk interventions extensively use rule-based decision-making. With long-term experience and training, these rules can be retrieved from the memory with little or no efforts [8]. Also, the growing presence of intraoperative sensors, e.g. endoscopic cameras, and representational information, e.g. on monitors, during the procedures makes the surgical training more difficult without the explicit understanding of the procedure along with the contextual awareness. In envisaged operation theatres [9], the efficacy of the operation will be achieved by addressing the workflow issues, but study participants also highlighted context awareness as an important functional requirement. The research also suggested that there is a lack of information on resources necessary to support surgical tasks and to efficiently plan the surgical process, which increases the surgical workflow variability and could be structured by grounding surgical process information in the ontology.

As a prototypical scenario, we chose Thoracentesis procedure. Pleural effusion is a life-threatening condition, often associated with other diseases, in which there is a collection of pathogenic or non-pathogenic fluids between the lung tissue and pleural space. Common symptoms of pleural effusion are pleuritic chest pain, coughing, and dyspnoea 
[10]. An invasive procedure, Thoracentesis, is performed for the removal of fluid from the pleural cavity. A needle is inserted into the chest cavity and the fluid withdrawn using a syringe [10]. Procedure-related complications account for at most $33 \%$ of people and are a major problem, and can range from pain, dry cough, no fluid return, or subcutaneous collection. Many life-threatening complications also arise due to surgical mishandling such as pneumothorax, pulmonary oedema, unintentional puncture of spleen or liver and sheared off catheter in the pleural space and in some exceptional conditions winging of the scapula [11]. Indeed, although Thoracentesis is a very simple procedure, procedure-related complications are higher than expected. A recent survey highlights gaps of knowledge and skills in conducting diagnostic and therapeutic Thoracentesis and shows significant training gaps as well [12]. Another survey [13] highlights that junior doctors were referring the majority of cases of Thoracentesis to be performed under the radiological guidance where the lack of experience accounts for $65 \%$ case referrals and the lack of supervision and guidance accounts for $49 \%$ of case referrals. The survey highlights junior doctors' deficiency in knowledge and cognitive procedural skills in performing Thoracentesis. Simulation-based training [14] and phantom model-based training [15] on Thoracentesis has found enhancing skills of the surgeons; however, the training systems were more focused on manual dexterity and lacked the aspects of context-awareness, intra-operative real-time guidance. Thus, there is indeed a need to create an intelligent training system.

We implemented an intelligent training system using knowledge-based system engineering. In this context, an ontology can be applied as a knowledge representation approach for process model that represents key concepts with their properties, relationships, and constraints for thoracentesis. An ontology provides a rich set of relationships between domain concepts, generally a set of 'part-of' or 'is-a' relations, and allows semantic rules to administer those relationships. In general, ontology holds all pertinent knowledge about the surgical procedure and can be represented in a computer interpretable format to reason over that knowledge to infer information on surgical tasks. Our approach for object recognition consists of the amalgamation of knowledge representation and sensor data to recognise surgical instruments in surgeon's hands for detecting surgical steps and on the scrub nurse surgical stand for further guidance on the required instrument in the next step of the surgical process. Knowledge representation and rule-based machine learning are involved in the object categorization process. Knowledge-based systems [16] [17] [18] have been used for object recognition by building a well-defined set of vocabularies for a domain of interest. Unfortunately, most of the algorithmic concepts in $3 \mathrm{D}$ computer vision are data-driven and recognition is mostly accomplished by describing the object's geometrical (roughness, curvature, for example) or physical features (colour, texture, for instance). Data-driven technologies heavily rely on algorithmic parameters and the object features itself. Moreover, these methods are highly static and few times do not achieve desired results in dynamic settings.

We previously developed [19] an ontology-based context-aware system framework for surgical assistance during Thoracentesis by using image processing and semantic technologies. The framework consisted of a Graphical User Interface (GUI), where user queries the surgical step, and then the queried step is forwarded to the component containing the ontology through Robot Operating System (ROS) [20] messages. Then the ontology component finds an instrument instance corresponding to the step of the surgery after the reasoning on the axioms specifying the surgical process model in the ontological assertion box through the description-logic based reasoners [21]. After recognising the instrument instance, the template-matching algorithm was used to recognise the instruments on the surgical table. In this paper, we also extended the knowledge-driven context aware framework [19] and included the dependencies, rules, and reasoning supporting the real-life activity of the surgeon. The system identified the contexts, e.g. surgical instrument, through a query to accomplish the surgical task, e.g. the requirement of a 50-ml syringe to withdraw the fluid from the chest cavity. The framework was not yet able to identify the surgical phases automatically, since the system did not have a robust inference mechanism to support the real-life activity. To overcome this limitation, we have included a rule-based inference mechanism, which improves the need of complex queries. We have also included an inference mechanism within the ontology to enhance the results of object recognition. In this article, we discuss the rule-based intelligent training system developed by using procedural knowledge stored in the ontology and recognising surgical instruments for surgical workflow understanding. Surgical workflow was constructed using the mutual influences between the surgical tasks. Therefore, each surgical task needs the results, e.g. information, on the earlier step and next instrument, from the previous surgical task as an input to proceed for the implementation of the workflow. The motivation of this research work is to develop a practical surgical training system that provides a low-level understanding of each surgical process task to trainee surgeons for accomplishing Thoracentesis. Eventually, the developed system will be helpful for automating Thoracentesis procedure, reducing iatrogenic surgical complications and improving patient care. 


\section{Materials and Methods}

\subsection{Context-aware software framework for intelligent training system}

The framework for intelligent training system mainly comprises three components to automatically derive information on the surgical workflow for surgical training and contextual awareness:

1. Knowledge module, where we implemented procedural knowledge on Thoracentesis in the form of an ontology and surgical workflow management through inference rules;

2. Computer vision module, where we implemented a) segmentation and tracking algorithms in the "segmentation node" and "tracking node" respectively to detect surgical instruments/materials using point cloud data acquired from two imaging sensors; and b) "markers node" - binary square fiducial markers for surgical instruments/materials recognition;

3. Data monitoring and Graphical User Interface (GUI), "MRS (Medical Robotics section of NeuroEngineering and medicAl Robotics Laboratory) context-aware system", where the system interacts with the clinicians and provides contextual information, e.g. instruments used in a particular phase of the surgical workflow.

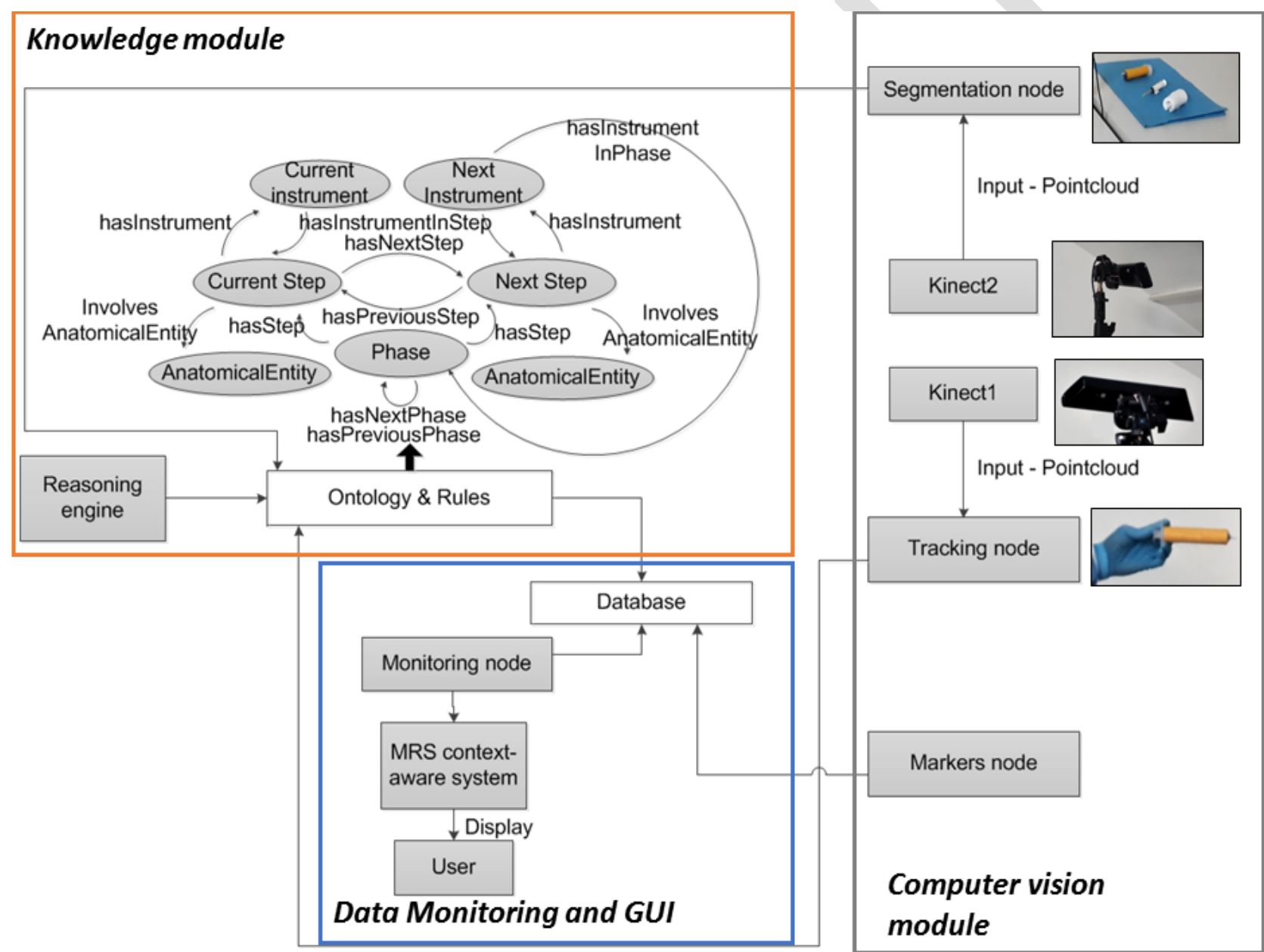

ROSJAVA AND SSH NETWORK

Fig. 1. The framework consists of 1) knowledge module, framed in orange colour, consisting of ontology, rule-base and Description Logic (DL), e.g. Pellet [21], based reasoning engine. Ontology is used to provide the controlled vocabularies that is used to represent the knowledge on Thoracentesis and its activities; and 2) Computer vision module, framed in grey colour, consisting of segmentation, marker recognition and tracking nodes. Our framework uses ROSJAVA, ROS messages and SSH (Secure Shell) network protocol to pass information between the knowledge module and the computer vision module and to facilitate the communication between two modules. 3) The data monitoring and GUI, "Monitoring node" and "MRS context-aware system", framed in blue colour, is used to do constant real-time monitoring of the database, e.g. populated based on the instruments' geometry and changes in the ontology, and to represent relevant information on surgical activities on the GUI. The overall system control is managed by the inference rules which used to a) control automatic workflow management b) recognise instruments in surgeon's hands and c) recognise instruments on the surgical stand. 


\subsection{Knowledge module}

\subsubsection{Ontology for Thoracentesis}

Ontology for Thoracentesis was built using a top-down approach, where most general concepts of the domain, such as phases (e.g. "Penetration") were first analysed and thereafter specialized concepts, such as actions (e.g. "WithDrawLargeSyringe"), were implemented. The needed information about Thoracentesis was obtained from a journal article [22], several online web resources, which were verified through HONCode [23] for health information authenticity and analysed using the method described in [24], and asking the opinion of a physician. The procedure was formalized using an approach like [25], where logical sentences were divided into triplets in the format of "Phase (Instrument, Step, Body Structure)", specified for each surgical phase, and surgical actions were linked to instruments and body structures. The developed ontology is based on an upper ontology (Basic Formal Ontology) [26]. Furthermore, we have integrated relevant parts of Foundational Model of Anatomy (FMA) [27], Information Artifact Ontology (IAO) [28] and W3C time ontology [29]. Theses ontologies are widely used in biomedical informatics and semantic web domain. We also identified several upper-level entities from OntoSPM ontology [30], an emerging common ontology for surgical process modelling, which also uses BFO and FMA for knowledge representation purposes. We extracted upper ontological entities using OntoFox tool [31].

The developed ontology is not only useful for contextual awareness but also to represent the full surgical workflow entities for Thoracentesis procedure. To create the surgical process model, we built production rules to map asserted facts in the knowledge base with the data obtained and features asserted online by the intra-operative sensors. We included features' values dynamically as the facts inside the ontology to recognise the relevant surgical phase. The result of situation interpretation is to identify the current step in which recognised instrument is being used and inferring the facts about next step and previous step based on the current step through the relations and activities grounded in the ontology. As a knowledge management tool for ontology, Protégé [32] version 5.0.0 was used.

\subsubsection{Production rules and surgical process modelling}

We implemented production rules to build the surgical process model. The implemented production rules were in the form of an implication between the antecedent (the statement comprised in "if" clause) and the consequent (the statement after "then" clause). The rules assume that the consequent actions, e.g. information on the step, are only executed if antecedent conditions, e.g. the recognition of the instrument, are satisfied. Rules are assumed to match ontology-based surgical knowledge with data from the sensors to infer knowledge on the surgical process model. As a conflict resolution strategy, which helps to choose which competing production rule to fire, rules, which already fired, were not considered for the recognition of phases. For example, " $50 \mathrm{~mL}$ syringe" is being used two times during "puncturing the chest wall" and during "withdrawal of fluids", however the rule execution is not considered once the rule for $50 \mathrm{~mL}$ syringe for puncturing the chest wall is executed. The next inference was achieved through the relationship between steps.

As shown in Fig. 2, we derived the basic rules for modelling surgical workflow of any surgical procedure that requires contextual awareness through ontology and image processing.

Rule syntax 1: Ontological reasoning with sensor data: "If 'instrument-and-instances' representing the realworld object and implicit description of object obtained through the image processing, then 'retrieveinstrument-and-its-model' information from the ontology". The rule was used to recognise instruments by geometric features extracted from the sensor and ontological knowledge.

Rule syntax 2: Ontological reasoning for the surgical process model: "If surgical step and instances of a recognized instrument is present then give information about current, previous, and next step of the procedure from the ontology". The rule was used to recognize the surgical activities.

Rule Syntax 3: Ontological reasoning with explicit information obtained to find implicit information: "If instances of a recognized instrument for the next step is present, then identify instrument model by segmenting the instrument from the surgical stand". The rule was used to recognize instrument from the real-world to be used in the next predicted surgical activity.

Fig. 2. Basic rules syntaxes that are required to accomplish the instrument recognition-driven automated surgical process model. It is assumed that rules are executed in the sequence of 1 to 3 for each surgical activity and anatomical locations are grounded explicitly in the ontology for each instrument. 
Fig. 3 represents example rules for the surgical workflow of the "Anaesthesia" phase of the procedure, where the system suggests the step based on the current instrument in surgeon's hand and suggest the next instrument required for the next step. "RULE-I" is used to recognise the instrument, e.g. "BetadineSwab", a material used to sterilise the skin of the chest, in surgeon's hand through a tracking algorithm as explained in section 2.4.1. "RULE-2" uses the already obtained information, based on "RULE-1", on instrument type to predict the status of the current surgical step and knowledge to accomplish the step e.g. the surgical actions. Further to that, the rule recognises predictive information on the previous and next step based on ontological constraints "hasPreviousStep" and "hasNextStep", respectively. The rule is also used to extract the information on the next instrument that is required in the next step. "RULE-3" recognises the instrument from the surgical stand that is required in the next step of the surgery, e.g. "Anaesthetise".

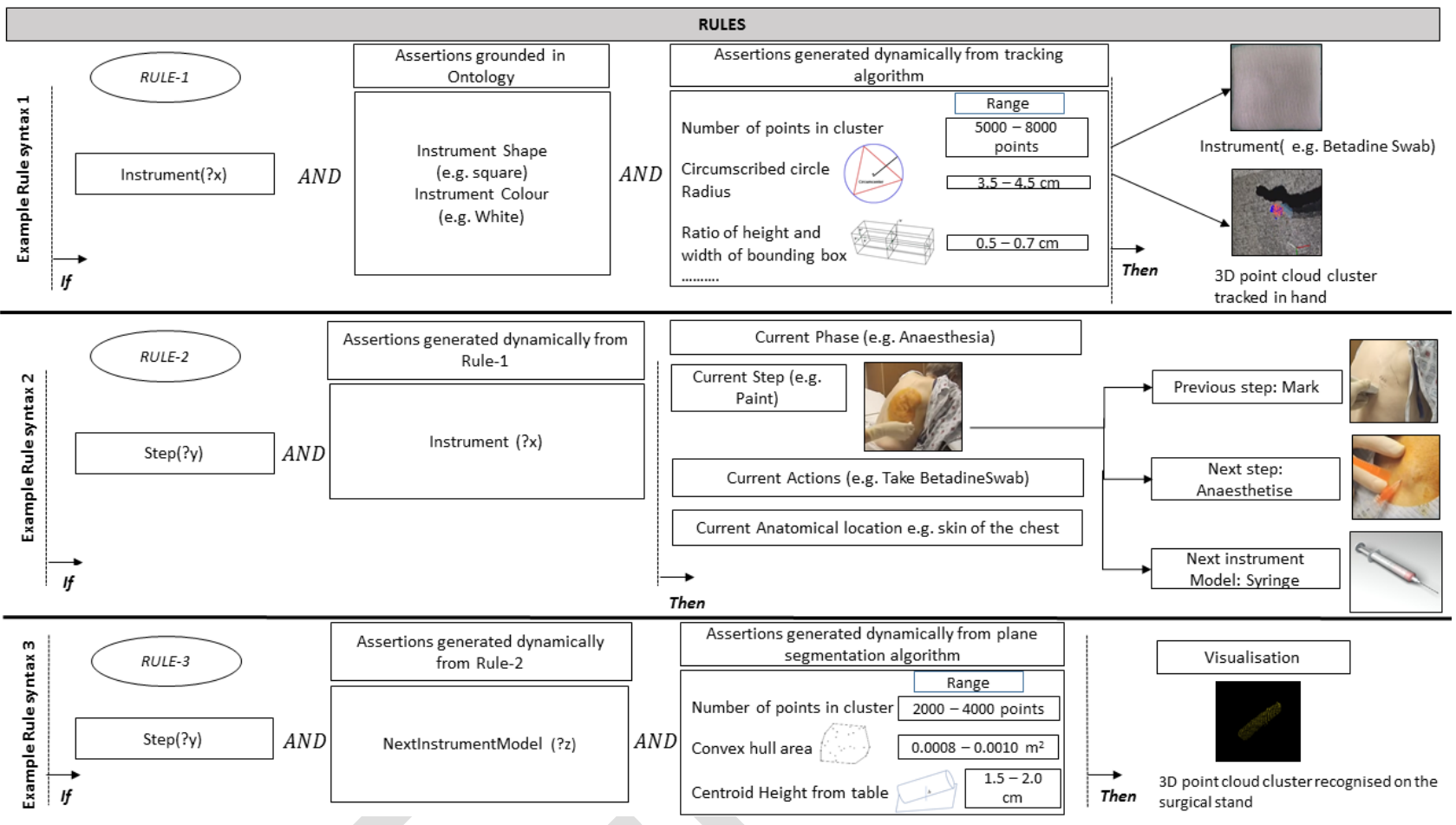

Fig. 3. A rule execution example for "Anaesthesia" phase of Thoracentesis for surgical training by contextual awareness

We used Semantic Web Rule Language (SWRL) [33] and Ontology Web Language - Description Logic (OWL-DL) [34], e.g. Pellet, to do rule-based deductive reasoning within the ontology to realise the surgical process model.

\subsection{Data acquisition framework}

We used imaging sensors, Microsoft Kinect for Xbox 360 (Microsoft, Redmond, WA, USA), for detecting the surgical tools in the scene. We acquired 3D point clouds, with a maximum acquisition frequency of $30 \mathrm{fps}$, to extract instruments' geometric features, for recognising surgical instruments in surgeon's hands (Kinect1) and on the surgical stand (Kinect2), as shown in Fig. 1. The data and relevant information were handled and stored using the non-SQL (Structured Query Language) based data storage on the file system, where concurrent access to the data files was established between two modules through SSH communication protocol, ROS, and with the help of Java NIO [35] to detect the changes in workflow entities, such as instruments, after retrieving from the ontology. Python Watchdog [36] library was used to constantly check the file system events to detect changes in the directories comprising the intelligent system database.

\subsection{Computer vision module}

There are two levels of abstraction for accomplishing surgical instruments recognition in the implemented framework for surgical training:

\subsubsection{Image processing level}

(1) We used particle filter to detect instruments in surgeon's hands [37], where outliers were removed with the help of statistical outlier removal [38] by analysing k-nearest neighbour distances for each point, and a bounding box around the 
tracked segment was created using Principal Component Analysis (PCA). Moreover, to optimize the algorithm, we used Kullback-Leiber distance sampling (KLD-sampling) [39] for adapting the size of the particle filters during the computation of the Kullback-Leibler distance (KL-distance) [39]. We extracted several features, such as (i) number of points in the cloud; (ii) the length of the instrument; (iii) ratio of height and width of the instruments; (iv) circumscribed circle between segment's centroid and the points at cloud's maximum and minimum $\mathrm{x}$-values. These features were used to recognise the surgical instruments in surgeon's hands.

For recognising the surgical instruments on the surgical stand, we used the RANSAC algorithm [40] [41], which is a widely-used computer vision technique to segment planes by estimating parameters of a mathematical models, e.g. the plane, from a set of data points. We down-sampled the points clouds, e.g. to $0.01 \mathrm{~m}$, and approximated the region of interest, with the help of voxel-grid and pass-through filters to decrease the point density and to remove the outlier points, such as walls [42], respectively. Conversely, we also used RANSAC-based plane segmentation to extract instruments' surface patches on the surgical stand. We extracted surface patch's (i) number of points in the cloud; (ii) instrument's centroid height from the plane, e.g. surgical stand; and (iii) area and volume of the convex hull representing instrument abstract size. We implemented particle filter and RANSAC-based plane segmentation in Point Cloud Library (PCL) [43].

(2) As shown in Fig. 4, we implemented ArUco markers [44] of size "20mm x 20mm" placed on each of the surgical instruments, where a separate "id" was assigned to each of these markers to successfully recognise the instrument. Each marker "id" corresponds to the same nomenclature as the ontological instances for instruments to be processed in contextaware system. Considering the limitation of Kinect resolution to detect four instruments e.g. three-wat stopcock, drainage tube and bag, $10 \mathrm{~mL}$ syringe, and flexible catheter, for the full Thoracentesis workflow and the training, we have recognised the instrument in surgeon's hands using ArUco makers. The ArUco markers were captured within $170^{\circ}$ field of view and the maximum distance between camera and markers was $50 \mathrm{~cm}$. We used 720p HD video stream input to detect the markers. The ArUco markers detection was implemented using OpenCV [45] library.

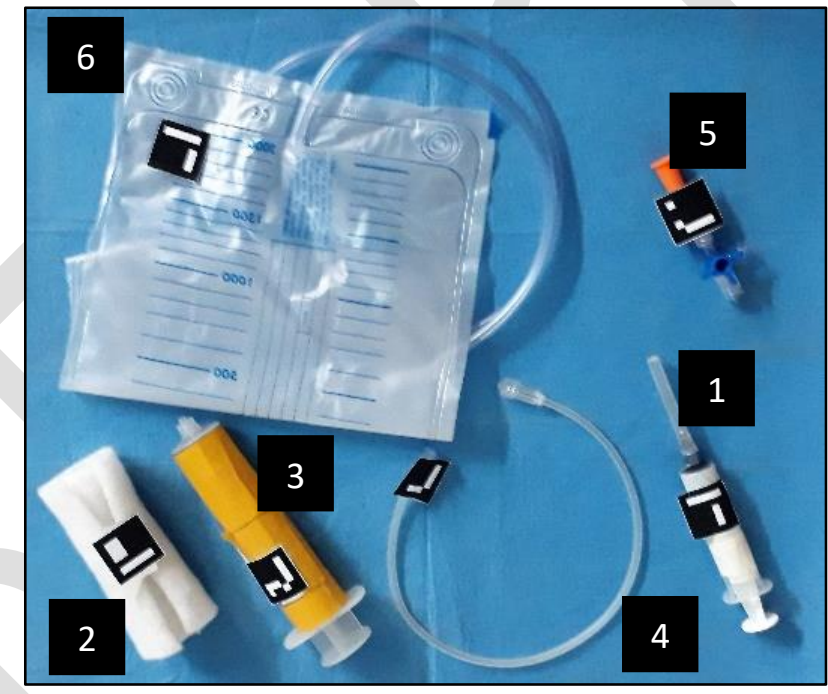

Fig. 4. ArUco markers was used to recognise surgical instruments/materials in surgeon's hands. $10 \mathrm{~mL}$ syringe (1) is used to anaesthetise the skin and superficial muscles of the chest region. Surgical swab (2), "betadine swab", is used to sterilise the skin and $50 \mathrm{~mL}$ syringe (3) is used to penetrate the intercostal muscles and the pleural cavity to withdraw the fluid. Flexible catheter (4) is used to connect $50 \mathrm{~mL}$ syringe with three-way stop-cock (5) and three-way stop-cock is used to change the channel of the withdrawn fluid e.g. to the 50-mL syringe and to the drainage bag (6). The drainage bag is used to collect the pleuritic fluid for further diagnostic examination.

\subsubsection{Symbolic data grounding and semantic recognition level}

(1) Symbolic data grounding level, where we extracted features' values as mentioned in section 2.4.1, e.g. "ratio of height and width of the instrument", and grounded each of the feature's values in the production rules within a certain range. We obtained values of the range by retrieving the geometric features from different view-points for each instrument through particle-filtering and RANSAC-based plane segmentation.

(2) Semantic recognition level, where instruments' feature values were mapped with the knowledge on the instrument dynamically grounded in the ontology through the semantic relations e.g. "hasRatio". 
We included a high-level information of instruments, such as the instrument colour is "white", and associated an instance of the instrument in the ontology with the 3D processed data, e.g. "hasCentroidHeightFromTable", obtained from an image sensor inside the ontology through the production rules to recognise surgical instruments and materials helpful to accomplish main tasks of Thoracentesis. Each extracted feature's value was published via ROS messages and added as values for the semantic relation, only, and only if, the values satisfied the constraints specified in the rules, to be processed by knowledge module. For example, the betadine swab is of approximately $1 \mathrm{~cm}$ height, where instrument's height value in "if" clause was specified using the range e.g. instrument's height is between $0.9 \mathrm{~cm}$ to $1.5 \mathrm{~cm}$, and assigned to specific semantic relation e.g. "hasCentroidHeightFromTable". Then the implicit information, e.g. height, of the instrument is obtained by computing instrument's centroid height from the surgical stand and dynamically included inside the ontology for instances of each surgical instrument. And "then" clause is specified the instrument as a "betadine swab" if the conditions in "if" clause matches with the implicit information dynamically grounded in the ontology.

\subsection{Data Monitoring and Graphical User Interface - MRS context-aware system}

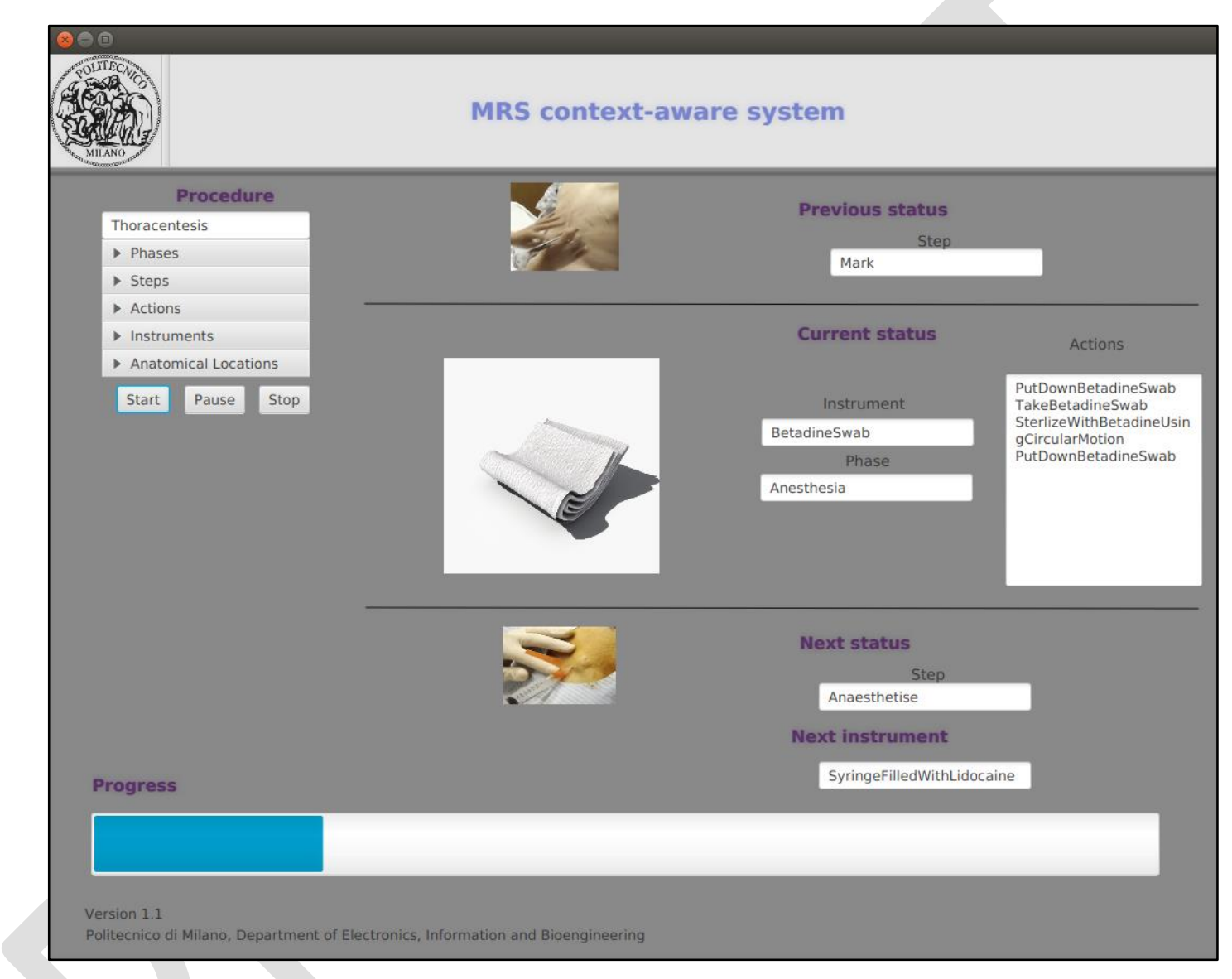

Fig. 5. The GUI provides interaction possibilities with the user, where the user can select the surgical procedure and see the different workflow entities, e.g. Phases, through the buttons in the menu on the left side of the GUI. The "Previous Status" represents the previous surgical workflow step, e.g. "Mark", that must have been performed before carrying out the activities specified in "Current Status". The "Current Status" represents the current phase, e.g. "Anaesthesia", of the surgery after detecting the instrument "BetadineSwab" and the surgical actions required to accomplish the specific step using the instrument. The GUI represents the "Next Status" displaying the next surgical workflow step, e.g. "Anaesthetise" and next instrument, e.g. "SyringeFilledWithLidocaine", required. The next instrument is also visualised, on a separate window, as a segmented surface patch from the surgical stand, recognised using the plane segmentation algorithm (Not shown in the figure). The step of the phase in the "Current Status" is not shown because the step is shown in the "Next Status" during the previous surgical activity for preventing the visualisation of the redundant information.

We developed a data monitoring system and GUI, "MRS context-aware system", as shown in Fig. 5, which constantly monitors the file system database for the changes in ontological instances retrieved by 3D image processing and production rules. While data monitoring interface updates the information online, the information is promptly available to the surgeons in the sterile environment with the help of GUI. The data monitoring system and the GUI were developed using JavaFX [46]. 


\section{Experimental Protocol}

The purpose of our experiments was to validate the ontology and the surgical process model, and to check the framework and the feasibility to use computer vision algorithms in assisting trainee users to learn the procedure knowledge and to understand the surgical workflow. Our experimental scenario was divided into three parts: (i) Ontology validation using the annotated surgical videos; (ii) instrument tracking and recognition in surgeon's hands and on the surgical stand; (iii) Naïve users training on Thoracentesis with pre-recorded videos and with the intelligent system.

\subsection{Ontology validation}

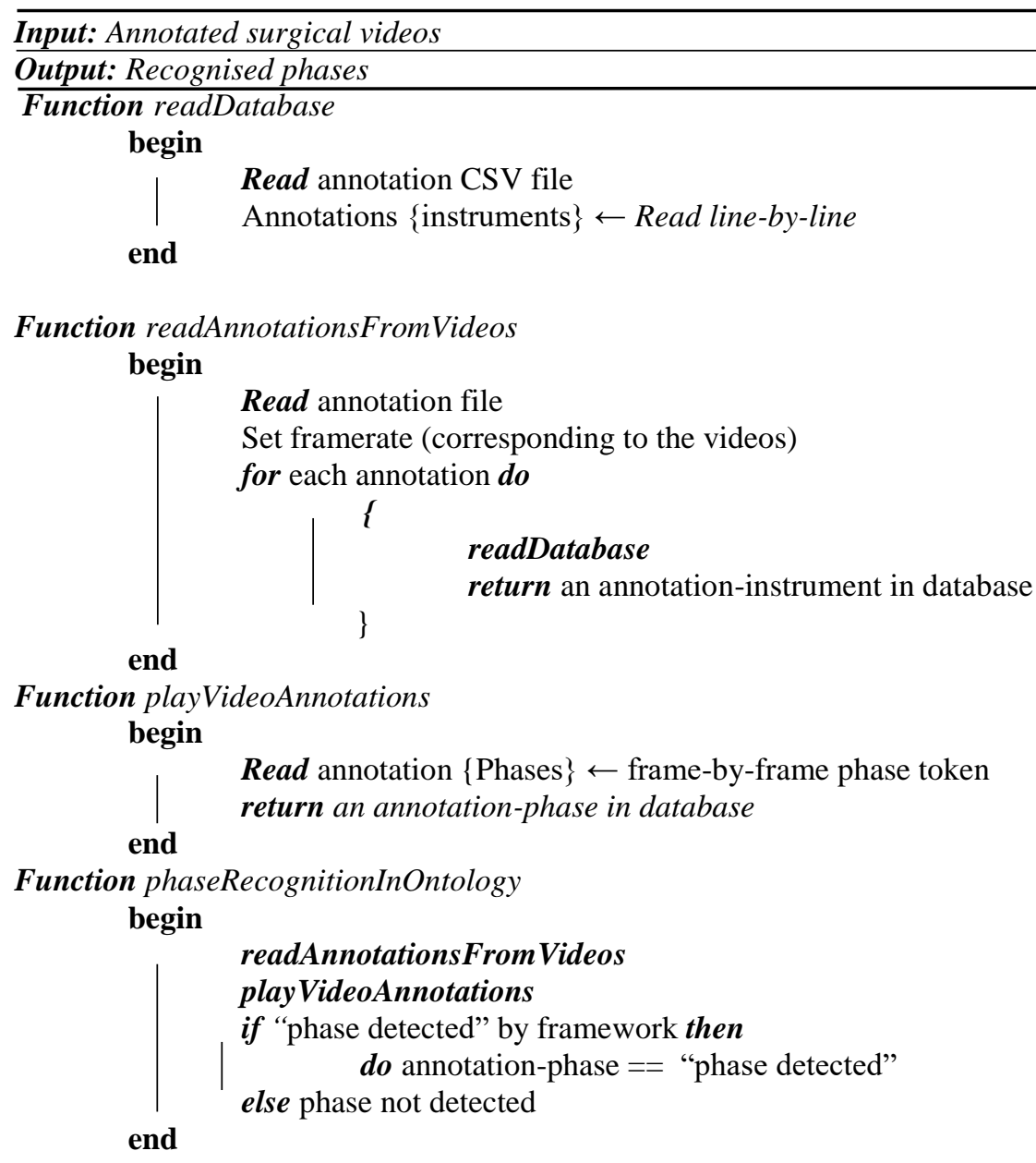

Fig. 6. The evaluation algorithm with video annotations for validation of ontology

We used video annotations data to validate the ontology. For annotating the controlled vocabularies specified in the ontology, which was used to specify surgical process model, we used a set of three videos on Thoracentesis from web resources, e.g. [22] [47] [48]. The videos were annotated frame by frame by two general physicians, with the mid-level expertise in performing Thoracentesis. We asked each clinician to annotate the "phase" of the surgery and an "instrument" in use during a specific instant of time. While annotating the videos, the audio was muted and required controlled vocabulary was explained to clinicians. We used Anvil annotation tool [49] for annotating the surgical videos.

As shown in Fig. 6, we extracted annotation files and saved them in the Comma Separated Value (CSV) format to be read by the framework components e.g. the knowledge module. The framework was provided only with the instrument annotations, as a context for current instrument in use in video, to recognise the surgical phases. We found the relative frequency, $R_{p}$, as shown in "Eq. (1)", of the inferred surgical phases through the framework with the ground truth phases annotated by the clinicians.

$R_{p}=\frac{P_{I}}{P_{T}}$ 
In Eq. "(1)", $P_{I}$ represents the number of correctly matched and classified phase instances and $P_{T}$ represents the total number of phase instances in annotations. We also measured the ontology classification time ( $\left.T_{\text {Onto }}\right)$ using Pellet [21] reasoner to measure the time required to classify the sub-sumption hierarchies in ontology before using it in the framework. The purpose of ontology validation was to confirm the satisfiability for Thoracentesis that the ontology covers all the entities and relationships require to represent knowledge on Thoracentesis. The video annotations were also used to analyse Thoracentesis to derive the requirements for segmentation and recognition algorithms. We analysed the annotations and found that surgeons were required to change the surgical instrument every approximately $1.8 \pm 0.6$ minute. The phases were changed every approximately $1.0 \pm 0.3$ minute.

\subsection{Instrument recognition in surgeon's hands and on the surgical stand}

To verify the system performance, we implemented a set of experiments using the set-up shown in Fig. 7. Considering the video annotations input, we tracked each instrument for approximately 2 minutes, i.e. 3600 frames. We fixed both sensor's viewpoint (Kinect 1 and Kinect 2 in Fig. 7) at approximately $45^{\circ}$ to get the maximum coverage of the surface of the instruments lying on the table. Before each experiment, we obtained an instrument surface patch by segmenting the nearest points, e.g. the points with the minimum depth values from camera frame, in the scene point-cloud, hereafter referred as "scene", by removing the plane (e.g. a top of the table) using RANSAC-based plane segmentation algorithm.

To check the feasibility of using the particle filter for tracking surgical instruments in surgeon's hands, we conducted five experiments with two naïve users (Fig. 9). We evaluated accuracy of the particle filter using cloud-tocloud Euclidean distance, "tracking error", $T_{e}$, using the shortest point distance by extracting the point clouds $\left(T_{i}\right)$ of the tracked instruments at every 30 frames. The we evaluated extracted point clouds with manually segmenting the instrument point-clouds, as a ground truth $\left(S_{i}\right)$, from the scene point clouds representing the same frame, by extracting the points that fall inside of a 2D polyline once it is projected on screen. We compared the two naive user's performances and two instruments tracking accuracy considering an empirical distance threshold of $0.01 \mathrm{~m}$.

$$
\left\|T_{e}\right\|=\sqrt{\sum_{i=1}^{K}\left(S_{i}-T_{i}\right)^{2}}
$$

In "Eq. (2)", $K$ represents the number of nearest neighbours, and $S_{i}$ and $T_{i}$ are the source and target points.

We correlated, using the Pearson correlation coefficient, the approximate actual time required to use the instruments with the time until the system tracked the instruments. To emulate the real scenario and instrument movements performed during Thoracentesis, we moved the hand in similar patterns during the experiments, where the surgeon holds the betadine swab in his/her hands. Further to that, the system recognises the surgical step and suggests next instrument on the surgical stand.

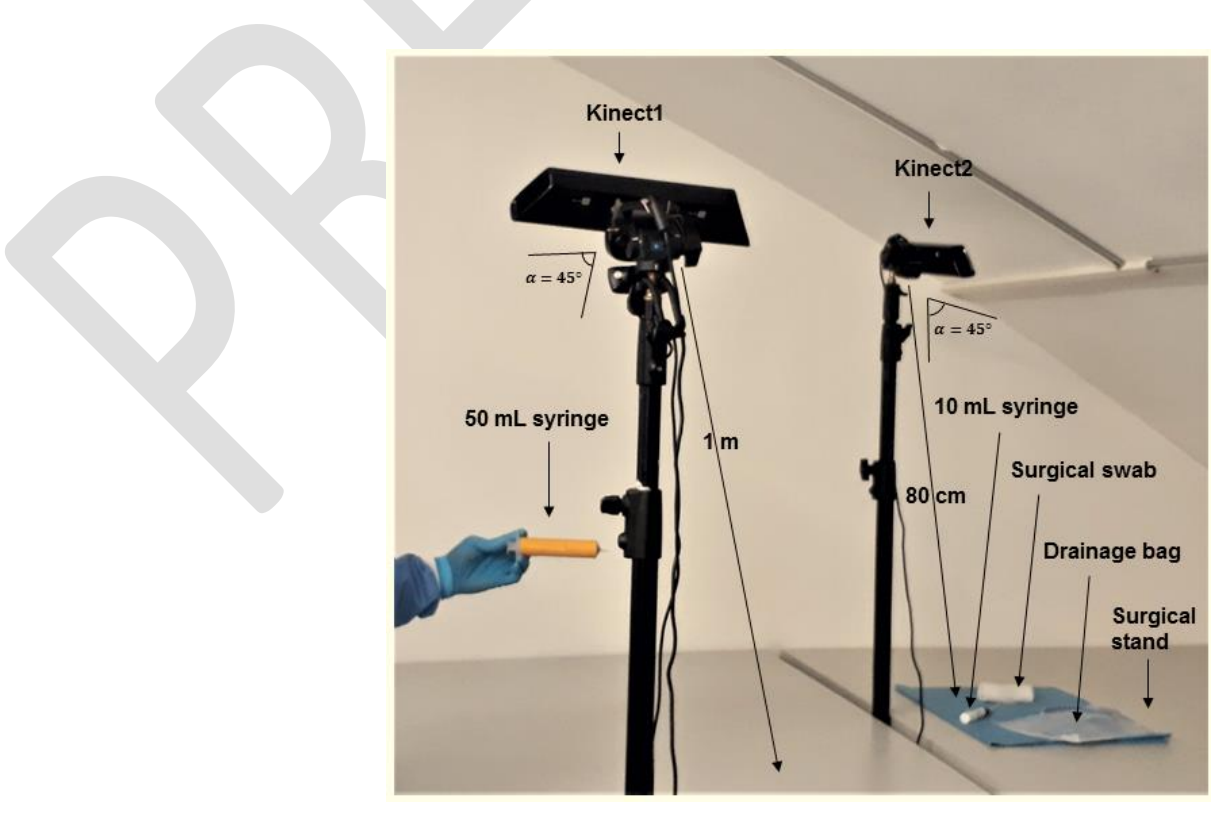

Fig. 7. The experimental setup includes Microsoft Kinects, the surgical stand and surgical materials and instruments. Kinect 1 for instrument detection using tracking was set approximately 1 meter away and Kinect 2 for object recognition on surgical stand was set 
approximately $80 \mathrm{~cm}$ away. The figure shows the surgical swab and the $50 \mathrm{~mL}$ syringe which were used during the experiments. Both the sensors were kept under the white fluorescent illumination or natural sun-light.

As the experiments were done in real-time, we repeated experiments for 25 times to report procedural classification time $\left(T_{P}\right)$, which was the time, the framework required to classify ontology for retrieving surgical workflow information, object recognition classification time $\left(T_{O}\right)$, which was the time, the framework required to classify ontology to recognise objects and system data synchronisation errors $\left(S_{E}\right)$, which was the errors incurred during the data monitoring.

To evaluate the instrument recognition on the surgical stand, we compared our result with the pure data-driven recognition algorithm, template-matching, implemented in our previous research [19]. We ran a total of 10 experiments for each instrument e.g. $10 \mathrm{~mL}$ syringe, surgical swab and $50 \mathrm{~mL}$ syringe. Each recognised instrument was verified by the instrument recognition result represented on the GUI. We also implemented a real-time interface which constantly displays the recognised instrument's surface patch obtained using plane segmentation.

\subsection{User experiments}

We evaluated the training system with 10 naïve participants, Biomedical Engineering graduate students at Politecnico di Milano, who were unware of Thoracentesis procedure. This study was carried out in accordance with the recommendations of our institute with written informed consent from the subjects in accordance with the declaration of Helsinki. The mean age of the participants were $24.2 \pm 2.3$ years. We divided participants into two groups of 5 participants each. Each group was taught Thoracentesis procedure with two different methods:

1) Group (1): Traditional mentor-based training, where a clinician taught Thoracentesis procedure by showing the procedures in three different videos and explaining the procedure concurrently. The clinical background for procedure was briefly explained to participants before starting the training;

2) Group (2): Intelligent system training, where participants were asked to hold the instruments of Thoracentesis, in the sequence of phases of Thoracentesis procedure as suggested by the system. The instruments were recognised as previously described in 2.4.1 to be used in the context-aware system to find the relevant contextual information, which was represented on GUI. The meaning of each ontological entity was explained only if asked by the participants. The clinical background for procedure was briefly explained to participants before starting the training.

After the experiments, both the groups' participants were asked to complete the questionnaire, as shown in Appendix A, which contained the questions about the procedural workflow and contexts, e.g. instrument requirements in different steps etc. We assessed the training efficiency by comparing the number of correct answers between the groups to evaluate their understanding and knowledge of the procedure and workflow. We also compared the time required to accomplish the surgical training and correct answers based on questionnaire evaluation between both the groups.

\section{Results and discussion}

In this section, we present the results of the individual components of the training system where the results follow the testing protocol mentioned in the section 3.

\subsection{Ontology}

The phase recognition results of our quantitative ontology validation, based on grouped video annotations are shown in Table 1.

\begin{tabular}{|l|l|l|l|}
\hline "Preparation" phase & "Anaesthesia" phase & "Penetration" phase & "Aspiration" phase \\
\hline $80 \%$ with $P_{T}=3$ & $100 \%$ with $P_{T}=5$ & $65 \%$ with $P_{T}=5$ & $100 \%$ with $P_{T}=5$ \\
\hline
\end{tabular}

Table 1. Surgery phase recognition results

Procedural knowledge on "Preparation" phase was inferred with $80 \%$ relative frequency $\left(R_{p}\right)$, while "Anaesthesia" phase was recognised with $100 \%$ relative frequency. The model did not infer the correct phases when the instances are repeated in annotations of consequent surgical activities. For example, $50 \mathrm{~mL}$ syringe is being used in two different phases of Thoracentesis, "Penetration" and "Aspiration". As there were repeated instances of $50 \mathrm{~mL}$ syringe for "Aspiration" and "Penetration" phase, i.e. "Penetration" phase was repeated again after "Aspiration" phase, the model inferred "Aspiration" phase incorrectly during "Penetration" phase. $R_{p}$ could be enhanced with the surgical training comprising the sequence 
of activities performed without repetition as we did for the user experiments. During Thoracentesis, "Aspiration" phase also lacks association with any anatomical structure, grounded as "NoAnatomicalLocation" in ontological assertion box for logical reasoning, which hinders the firing rules for accurately detecting the "Penetration" phase, when the phase is represented again. The ontology classification time for class hierarchies $\left(T_{\text {onto }}\right)$ was $357 \mathrm{~ms}$.

\subsection{Instrument recognition}

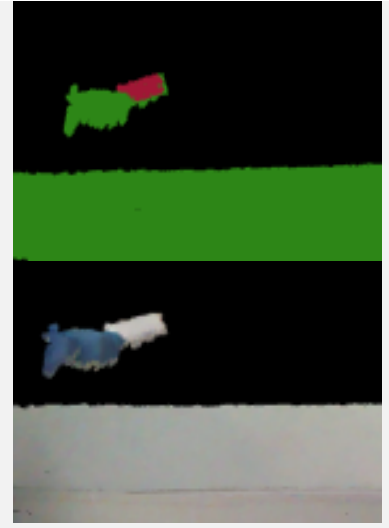

00:00:09s

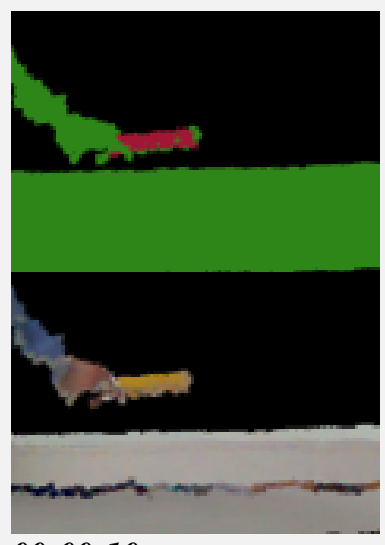

00:00:10 $s$

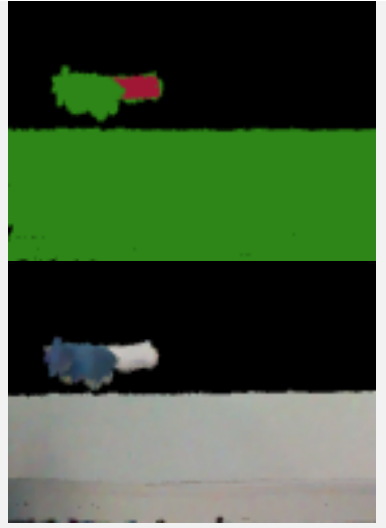

$00: \overline{00: 16} s$

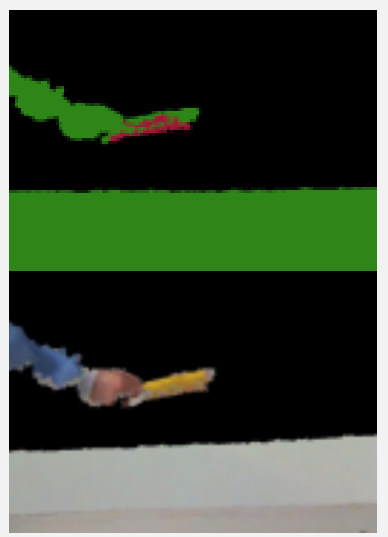

00:00:28 s

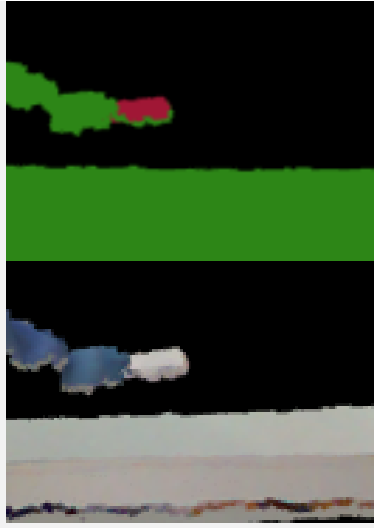

00:00:24 s

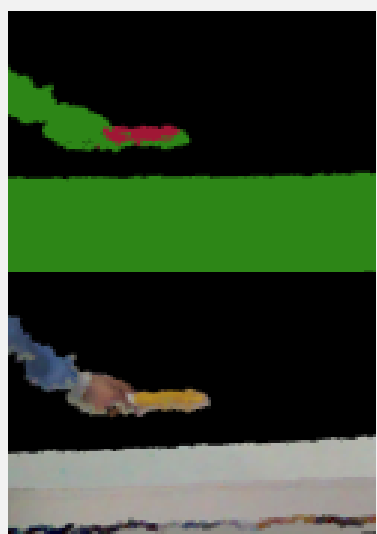

$00: 00: 42 s$

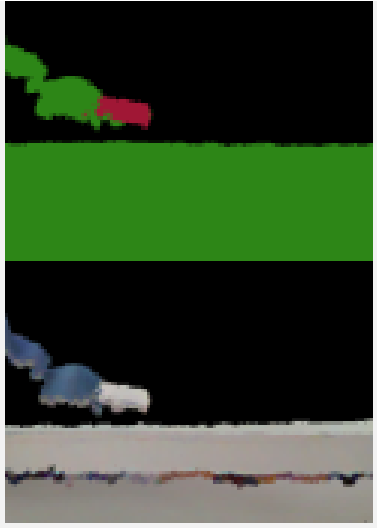

00:00:70 $s$

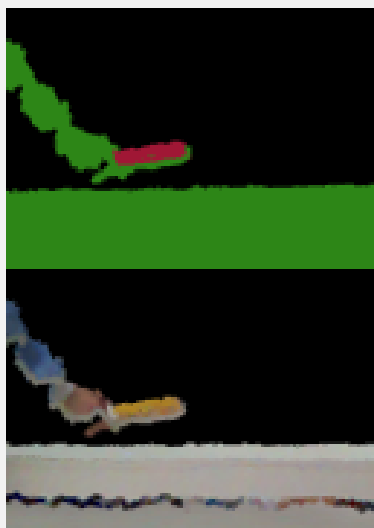

00:00:80 s

Fig. 8. Tracking results on surgical swab (in the top row) with partial occlusions and $50 \mathrm{~mL}$ syringe (in the bottom row without gloves) sequences. The red points represent tracked surgical instrument cluster

As shown in Fig. 8, we were able to successfully track surgical swab and $50 \mathrm{~mL}$ syringe until 4 minutes, then we stopped tracking it, which is satisfactory, as shown in Table 2, considering the time required to change the instruments based on video annotations. The system was not being able to track $10 \mathrm{~mL}$ syringe because of its small dimension, but this is a problem with the sensor resolution. As we also performed the experiments with down-sampled point clouds, to increase the speed of algorithm execution, the sample size e.g. segmented instrument cluster size, e.g. $112 \pm 53$ as shown in Table 2, was much smaller to initialize the tracking algorithm for $10 \mathrm{~mL}$ syringe. Further to that, we found that up-sampling of point clouds does not affect tracking results for the above mentioned surgical instruments e.g. $50 \mathrm{~mL}$ and $10 \mathrm{~mL}$ syringes and materials e.g. surgical swab.

\begin{tabular}{|l|l|l|l|}
\hline Instrument/Material & $\begin{array}{l}\text { Instrument cluster } \\
\text { number of points }\end{array}$ & $\begin{array}{l}\text { Time (s) (instrument } \\
\text { usage time based on video } \\
\text { annotations) }\end{array}$ & $\begin{array}{l}\text { Time (s) (based on system } \\
\text { tracking) }\end{array}$ \\
\hline $50 \mathrm{~mL}$ syringe & $939 \pm 71.4$ & $26.8 \pm 10.5$ & 240 \\
\hline Surgical swab & $1006 \pm 76.02$ & $12.2 \pm 1.8$ & 240 \\
\hline $10 \mathrm{~mL}$ syringe & $112 \pm 53$ & $26.8 \pm 4.8$ & 0 (Not tracked) \\
\hline
\end{tabular}

Table 2. The time until the system tracked the instruments and the actual approximate time required to track instruments during the procedure 
Tracking results

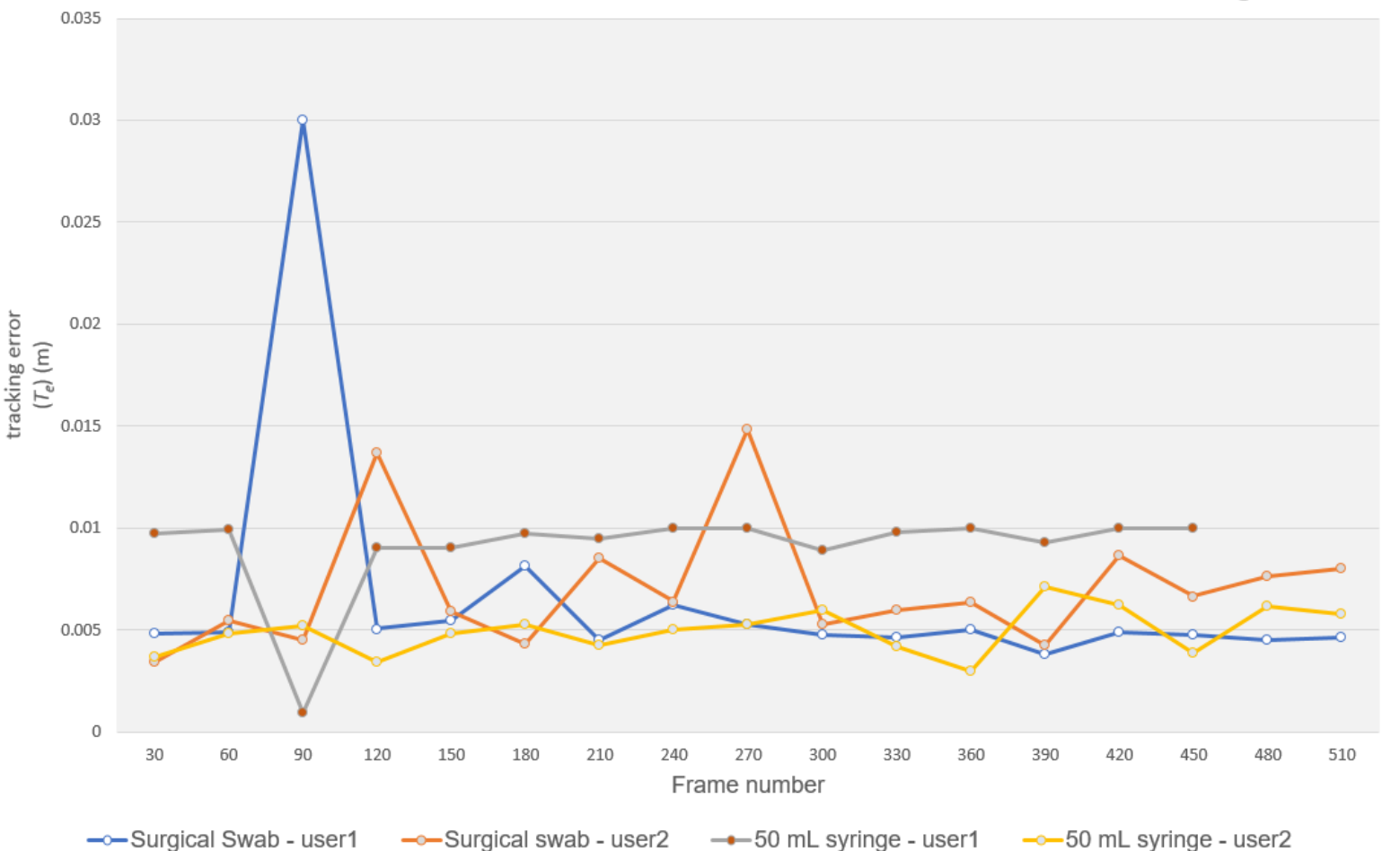

Fig. 9. The chart represents the tracking error during tracking of the instruments, e.g. (a) surgical swab and (b) $50 \mathrm{~mL}$ syringe in surgeon's hand, wearing gloves, during the surgical tasks of Thoracentesis

As shown in Fig. 9, The system was able to track surgical swab and $50 \mathrm{~mL}$ syringe for 510 frames (around 4 minutes), around 2.13 seconds for processing each frame. Considering an empirical tracking error threshold of $0.01 \mathrm{~m}$ based on visual inspection, the tracker lost the tracking for Surgical Swab for three times, at frame 90 for user1 and frames 120 and 270 for user2, due to unexpected hand motions but recovered immediately. We were able to track surgical swab and $50 \mathrm{~mL}$ syringe with $88.23 \%$ and $100 \%$ accuracy respectively based on the tracking threshold. The algorithm was not able to track other instruments and materials due to inappropriate instrument surface patches representing partial surface point-clouds. As mentioned in section 2.4.1, we were able to recognise all the instruments with $100 \%$ accuracy with ArUco-marker based recognition.

In our previous work [19], we used the template-matching to recognise the surgical instruments on the surgical stand. During our experiments with static point-cloud scene datasets, we achieved $93.3 \%$ accuracy in determining $50 \mathrm{~mL}$ syringe. The template matching had very high false positive results, as much as $80 \%$, for $10 \mathrm{~mL}$ syringe and surgical swab. In our current marker-less scenario experiments for context awareness, we recognised $10 \mathrm{~mL}$ syringe, $50 \mathrm{~mL}$ syringe and surgical swab with $90 \%$ accuracy. The algorithm failed to recognise the instrument only when there was a fault in segmentation of surface patches, which was found to be the segmentation of outlier points. e.g. non-instrument cluster.

As shown in Fig. 10, the classification time was higher at the beginning of the experiments, e.g. an iteration contains a full cycle of instrument recognition and phase identification, but it was gradually improved because initial iterations require population of file system database, which also to be represented by the GUI to unfold the contextual information. We measured Pearson correlation coefficient $(\mathrm{R}=0.9918$, " $\mathrm{p}<0.05$ "). There is a strong correlation between the time required to recognise the instrument with the time required to infer the procedural knowledge and contextual information (or vice versa). During our experiments, we found data synchronisation errors $\left(S_{E}\right)$ during concurrent access to the file system. The main reason was systematic Java error to read the dynamically updating byte streams information to replace the recognised contextual information in file database. The systemic error could have been resolved using the relational database structures. 
Full version at: http://www.sciencedirect.com/science/article/pii/S0933365717301525

https://doi.org/10.1016/j.artmed.2017.10.004

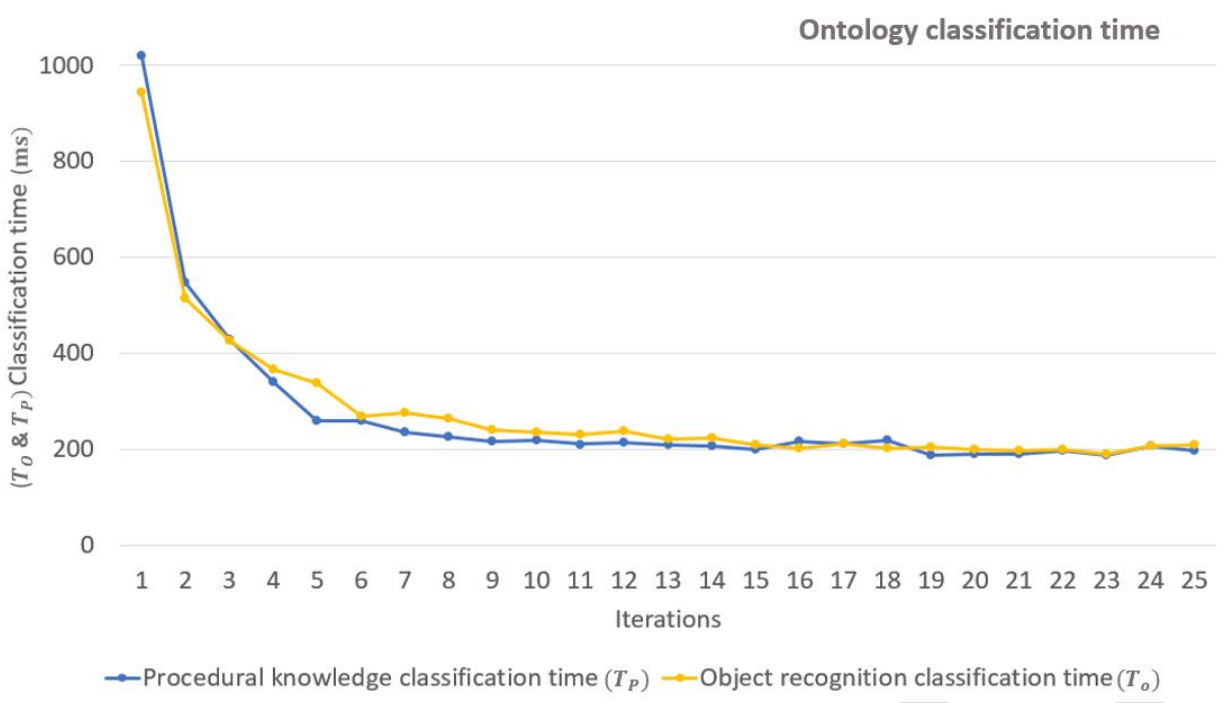

Fig. 10. The figure represents the time required to classify the ontology to infer the instances that contain the contextual information, e.g. instrument recognition in scene, mainly the time, "the object recognition classification time" $\left(T_{O}\right)$, required to compare dynamically retrieved instrument geometric information, e.g. features' values, with production rules to recognise the instrument (yellow line); and after the recognition of an instrument, the time, "the procedural knowledge classification time" $\left(T_{P}\right)$, required to classify the ontology for recognising surgical phases and related information (blue line).

\subsection{User evaluation}

As shown in Fig. 11, while the participants with traditional mentor-based training gave $6.4 \pm 0.96$ correct answers on procedure knowledge, the participants who learned the procedure with our approach gave $7.5 \pm 1.58$ correct answers.

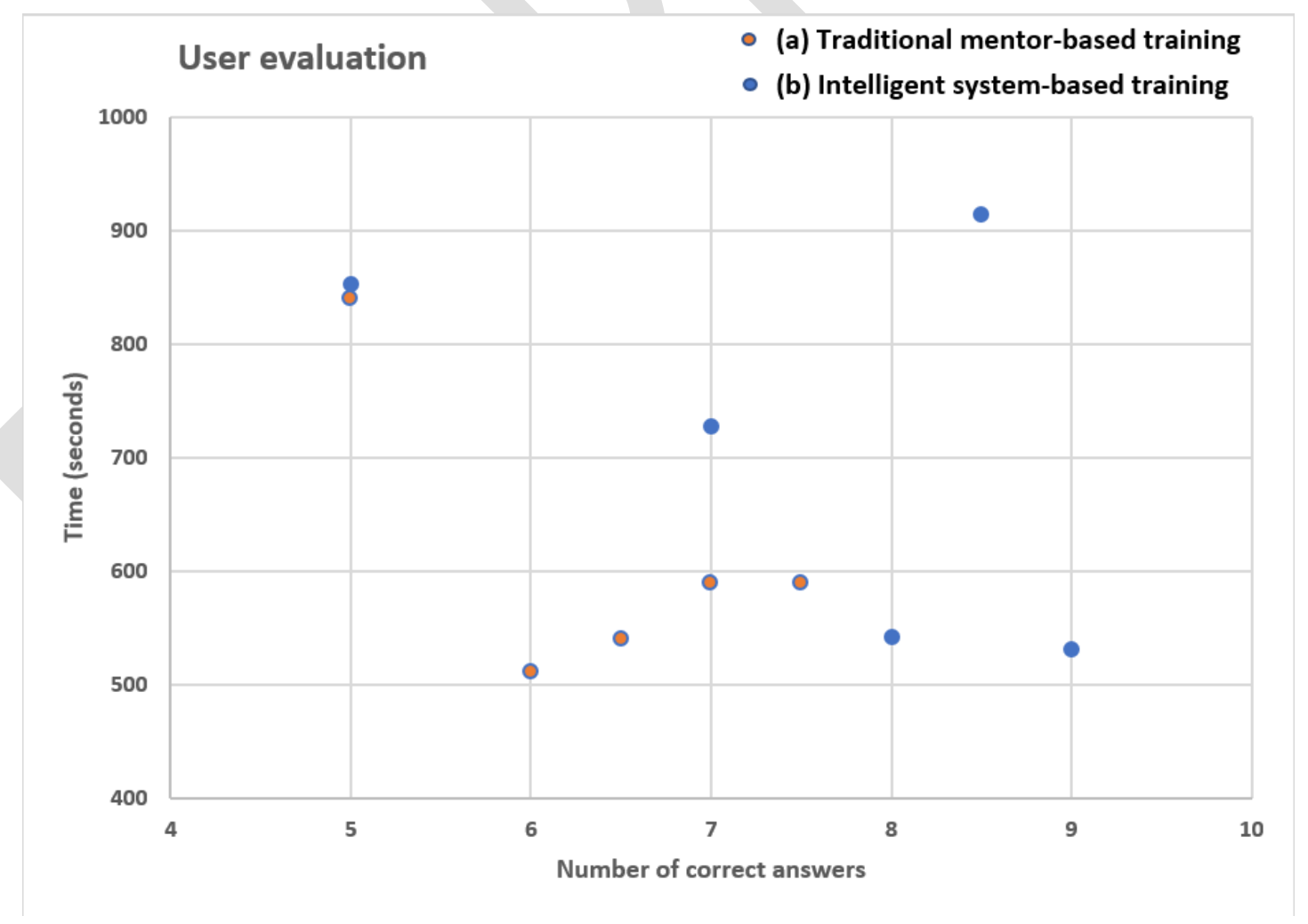

Fig. 11. The chart represents (a) video-based (5 participants) and (b) system-based (5 participants) experiments of Thoracentesis training, showing the number of correct answers and the time required to complete the training 
There was also no relationship between the number of correct answers, e.g. understanding of the procedure, and the time a participant took to finish the training (" $r=-0.65$ " with " $p<0.05$ " for video-based training and " $r=-0.46$ " with " $p<0.05$ " for intelligent system-based training).

The qualitative user feedback with intelligent system-based training suggested that it was difficult to follow the number of contextual information represented on GUI. Moreover, most of users were only following the instructions, esp. representative figures and surgical actions, represented on GUI during the system-based training, which proves to lower the cognitive load, e.g. thinking, with the structured representation of surgical information. The users also suggested that pictorial representation would be more helpful than text-based representation of surgical contexts.

\section{Conclusion}

We presented an intelligent training system for Thoracentesis, which we implemented using context-aware software framework for learning the procedure. We implemented an ontology with inference rules and recognised surgical instruments to make an instrument-recognition driven surgical process training, considering the anatomical locations are already grounded for each surgical activity. The proposed training system aids the cognitive phase of the surgical training [50], where users read, watch and intellectualize the individual surgical activities. Our approach allows trainees to accurately assess their own understanding of the procedure and recognise what the new surgical activity would be, for constructing a mental model, based on the identified activity on the surgical phases represented by the system. While the current surgical training systems still lack the full cognitive support, the represented work is an important step to introduce a new paradigm for the surgical training. Other potential benefit is the modular design of the context-aware system framework components, which could be also replicated for advanced robot assisted surgery system architectures for cognitive guidance. We researched a new possible set of syntaxes, serialised using SWRL rules that could be helpful with proposed knowledge representation for surgical phase recognition after recognition of instruments. While the previous study was done with the simulation-based setting [51], where ontology and inference rules were used as surgical workflow modelling, the developed context-aware system framework allowed real-time processing of data and possibility to extend the framework due to its modular approach.

The major study limitation was recognition of instruments with Kinect sensor, which could have provided to use the system in marker-less setup. The "anatomical location" were grounded in ontology, while combined recognition of anatomical location and instrument would enhance the results increasing the potential of framework to be used for complex surgeries e.g. laparoscopic surgeries. As explained in section 4.1, system would fail with the repeated instances, thus the currently developed model failed to capture the surgical variability. The data synchronisation errors prove that the system components still fail to maintain transaction consistency, which may be solved by the stream reasoning on ontology e.g. C-SPARQL [52].

As future work, the current system could help retrieving individual surgeon's skills profile as an individual ontology which represents the inferred phase and instruments usage while performing the procedure. Eventually these ontologies could be useful to analyse the surgical skills and user performance. We propose creating new likelihood functions for particle filtering, generating the automated workflow rules using inductive learning, e.g. First-Order Inductive Learner [53] [54] to create individual disease- [55] and procedure-specific [51] surgical process models and testing the system in real-surgical environments where the system retrieves the contextual information to trainees based on the expert performing the procedure. The context-aware system framework could further be enhanced to be used for intention detection [56] in human-robot interaction and for automating and planning [57] the robot-assisted surgeries, which will be eventually used in SMARTsurg project as to develop advanced cognition and perception abilities. SMARTsurg project will develop an advanced system for performing Robot Assisted Minimally Invasive Surgery (RAMIS) to reduce surgeon's cognitive load to shorten the training time and to deliver accuracy, safety, reduced procedure time and expanded applicability.

\section{Acknowledgement}

Funding: This project has received funding from the European Union's Horizon 2020 research and innovation programme under grant agreement No. H2020-ICT-2016-732515.

\section{References}

1. Moulton C-A, Dubrowski A, MacRae H, Graham B, Grober E, Reznick R. (2006) Teaching surgical skills: What kind of practice makes perfect? Annals of Surgery, 244(3), 400-409. 
2. Baldwin P.J., Paisley A.M., Brown S.P. (1999) Consultant surgeons' opinions of the skills required of basic surgical trainees. British Journal of Surgery, 86, 1078-82.

3. Flin R.H., O'connor P, Crichton M. (2008) Safety at the sharp end: a guide to non-technical skills. Aldershot, England; Burlington, VT, Ashgate.

4. Driscoll P.J., Paisley A.M., Paterson-Brown S. (2003) Trainee's opinions of the skills required of basic surgical trainees. The American Journal of Surgery, 186, 77-80.

5. Zhang L, Kamaly L, Luthra P, Whitfield P. (2016) Simulation in neurosurgical training: a blueprint and national approach to implementation for initial years trainees. British Journal of Neurosurgery, 30(5), 577-81.

6. McSparron J.L., Michaud G.C., Gordan P.L., Channick C.L., Wahidi M.M., Yarmus L.B., Feller-Kopman D.J., Makani S.S., Koenig S.J., Mayo P.H., Kovitz K.L., Thomson C.C. (2015) Simulation for skilled-based education in pulmonary and critical care medicine. Annals of the American Thoracic Society, 12(4), 579-86.

7. Scottish Audit of Surgical Mortality. Annual Report. 2010. http://www.sasm.org.uk/Publications/Main.html [Online]. Accessed 20.02.2017.

8. Flin R, Youngson G, Yule S (2007) How do surgeons make intraoperative decisions? Qual Saf Health Care, 16(3), 235-39.

9. Cleary K, Kinsella A, Mun S.K. (2005) OR2020 workshop report: operating room of the future, In: Lemke HU, Inamura K, Doi K, Vannier M.W., Farman A.G., editors. CARS 2005: Proceedings of the $19^{\text {th }}$ International Conference on Computer Assisted Radiology and Surgery; Berlin, Germany, 832 -838.

10. Broaddus C, Light R. (2010) Murrey and Nadel's textbook of respiratory medicine, $5^{\text {th }}$ edition (Saunders Elsevier, Philadelphia).

11. Faruqi S, Raychaudhari C, Thirumaran M, and Blaxill P. (2009) Winging of the scapula: an unusual complication of needle thoracentesis. European Journal of Internal Medicine, 19(5), 381-382.

12. Porcel J.M., Cases-Viedma E, Bielsa S (2016) A survey to medical residents on the performance of diagnostic and therapeutic thoracentesis: a training gap? Revista clinica espanola, 216(9), 474-480

13. Aiyappan V, Munawar A, Thien F (2013) Junior doctor training in pleural procedures: a quality survey. Intern Med J, 43(1), $96-100$.

14. Barsuk J.H., Cohen E.R., Williams M.V., Scher J, Feinglass J, McGaghie W.C., O’Hara K, Wayne D.B. (2016) The effect of simulation-based mastery learning on thoracentesis referral patterns. Journal of Hospital Medicine, 11 (11), 792 -795 .

15. Wojtczak J.A. (2014) Models to teach lung sonopathology and ultrasound-guided thoracentesis. Journal of Ultrasonography, 14(59), 367-70.

16. Maillot N.E., Thonnat M (2008) Ontology based complex object recognition. Image and Vision Computing, 26 (1), 102-113.

17. Liu S, Thonnat M, Berthod M (1994) Automatic classification of planktonic foraminifera by a knowledge based system. In: (Eds) The tenth conference on Artificial Intelligence for Applications, IEEE Computer society press, San Antonio, Texas, 358-364.

18. Hmida H.B., Boochs F, Cruz C, Nicolle C. (2012) Knowledge base approach for 3D object detection in point clouds using $3 \mathrm{D}$ processing and specialists knowledge. International Journal of advances in intelligent systems, 5(1 \& 2), 1-14.

19. Nakawala H, Ferrigno G, De Momi E. (2017) Toward a knowledge-driven context aware system for surgical assistance. Journal of Medical Robotics Research, 2 (3), 1740007. (In press)

20. Quingley M, Conley K, Gerkey B, Faust J, Foote T, Leibs J, Wheeler R, Ng Andrew (2009) ROS: an open-source Robot Operating system. In: ICRA workshop on open software, 3 (3.2), 5.

21. Evren S, Parsia B, Cuenca Grau B, Kalyanpur A and Katz Y. (2007) Pellet: A practical OWL-DL reasoner, Web Semantics: Science, Services and Agents on the World Wide Web, 5 (2), 51-53. 
22. Thomsen T.W., DeLaPena J, and Setnik G.S. (2008) Thoracentesis Videos in clinical medicine. The New England Journal of Medicine, 355(15), e16.

23. Health On the Net Code of Conduct - Health On Net Foundation, Switzerland. Available from: https://www.healthonnet.orhg/HONcode [online] Accessed 17.10.2016.

24. Natalya N. F., and McGuinness D.L. (2001) Ontology development 101: A guide to creating your first ontology, Stanford knowledge systems laboratory technical reports KSL-01-05 and Stanford medical informatics technical reports SMI-2001-0880.

25. Neumuth T, Strauß G, Meixensberger J, Lemke H.U., and Burgert O. (2006) Acquisition of process descriptions from surgical interventions In DEXA 2006 LNCS, vol. 4080, Springer, S. Bressan, J. Kung, R. Wagner (Heidelberg, Germany), 602-611.

26. Grenon P and Smith B. (2004) SNAP and SPAN: Towards Dynamic Spatial Ontology. Spatial Cognition and Computation, 4:1, 69-103.

27. Rosse C and Mejino J.L.V. (2007) The Foundational Model of Anatomy Ontology, in Burger, A and Davidson, D and Baldock, R, Eds. Anatomy Ontologies for Bioinformatics: Principles and Practice, pp. 59-117.

28. Information Artifact Ontology. https://code.google.com/p/information-artifact-ontology [Online]. Accessed 17.08.2016

29. W3C Time Ontology https://www.w3.org/TR/owl-time/ [Online]. Accessed 20.08.2016.

30. Gibaud B, Penet C, Jannin P. (2014) OntoSPM: a core ontology of surgical procedure models, SURGETICA.

31. Xiang Z, Courtot M, Brinkman R.R., Ruttenberg A and He Y. (2010) OntoFox: web-based support for ontology reuse, BMC Research notes 3:175.

32. Protége 2016 Jan Stanford Center for Biomedical Informatics Research. Available from: http://protege.stanford.edu [Online] Accessed 12.01.2016.

33. Horrocks I, Patel-Scheider P, Boley H, Tabet S, Grosof B, Dean M. SWRL: A Semantic Web Rule Langauge combining OWL and RuleML. W3C Member Submission 2004, https://www.w3.org/Submission/SWRL [Online]. Accessed 23.02.2017.

34. Horridge M, Bechhofer S. (2011) The OWL API: A Java API for OWL Ontologies. Semantic Web, 2 (1), 11-21.

35. Leonard A, Watch Service API, Pro Java 7 NIO.2, (2011), Apress, pp. 111-134.

36. Beazley D, Jones B.K. Python Cookbook, 3rd edition, Recipes for Mastering Python 3, (2013), O’Reilly Media.

37. Dellaert F, Burgard W, Fox D, and Thrun S. (1999) Using the condensation algorithm for robust, vision-based mobile robot localization. In proceedings of IEEE Computer Society Conference on Computer Vision and Pattern Recognition,

38. Rusu R.B., Marton Z.C., Blodow N, Dolha M, Beetz M, Towards 3D point cloud based object maps for household environments, Robotics and Autonomous Systems, 56 (11), 927-941.

39. Fox, D (2001) KLD-sampling: Adaptive particle filters. Advances in neural information processing systems, Cambridge, MA, 713-720.

40. Schnabel R, Wahl R, Klein R (2007) Efficient RANSAC for point cloud shape detection. Computer Graphics Forum, 26(2), 214-226.

41. Fischler A.M. and Bolles C.R. (1981) Random sample consensus: a paradigm for model fitting with applications to image analysis and automated cartography, Communications of the ACM. 24(6) (1981), 381-395.

42. Gupta M, and Sukhatme G. (2012) Using manipulation primitives for brick sorting in clutter, Robotics and Automation (ICRA), IEEE International Conference On, Saint Paul, Minnesota, USA, 3883-3889.

43. Rusu R.B., and Cousins S. (2011) 3D is here: Point Cloud Library, in: IEEE International Conference on Robotics and Automation, $1-4$. 
44. Garrido-Jurado S, Muñoz-Salinas R, Madrid-Cuevas F.J., and Marín-Jiménez M.J. (2014) Automatic generation and detection of highly reliable fiducial markers under occlusion. Pattern Recogn, 47(6), 2280-2292. DOI=10.1016/j.patcog.2014.01.005

45. Bradski G, (2000), opencv_library, Dr. Dobb's Journal of Software Tools, 4.

46. Anderson G, and Anderson P. (2009) Essential JavaFX. Pearson Education.

47. PACES MRCP UK resources (2009, Jun 7), “Thoracentesis" [video file], Retrieved from https://www.youtube.com/watch?v=2ZRip1STSSQ\&t=74s.

48. InfoTel-Med (2009, Mar 7), “Toracocentesis" [video file], Retrieved from https://www.youtube.com/watch?v=xgmP4F6aR3Q.

49. Kipp M.,"Spatiotemporal Coding in ANVIL," Proc. of the 6th international conference on Language Resources and Evaluation (LREC-08), pp. 2042-2045, 5/2008, Marrakech, Morocco.

50. Chatterjee S, Ng J, Matsumoto E.D. (2009) Assessing the surgical decision making ability of novice and proficient urologist, 181 (5), 2251-2260.

51. Katić D, Julliard C, Wekerle A.-L., Kenngott H., Müller-Stich B.P., Dillmann R, Speidel S, Jannin P, Gibaud B, (2015) LapOntoSPM: an ontology for laparoscopic surgeries and its application to surgical phase recognition, International Journal of Computer Assisted Radiology and Surgery, 8, 1427-34.

52. Berbieri D.F., Braga D, Ceri S, Valle E.D., Grossniklaus M. (2010) Querying RDF streams with C-SPARQL, SIGMOD Record, 39(1), 20 - 26.

53. Quinlan J.R. (1990) Learning logical definitions from relations, Machine Learning, 5 (3), 239-266.

54. Nakawala H, De Momi E, Pescatori L.E., Morelli A, Ferrigno G. (2017) Inductive learning of the surgical workflow model through video annotations, 30 $0^{\text {th }}$ IEEE International Symposium on Computer-Based Medical Systems, Thessaloniki, Greece (Submitted).

55. Kassahun Y, Perrone R, De Momi E, Berghöfer E, Tassi L, Canevini M.P., Spreafico R, Ferrigno G and Kirchner F. (2014) Automatic classification of epilepsy types using ontology-based and genetic-based machine learning, Artif. Intell. Med, 61(2), 79-88.

56. Nessi F, Beretta E, Gatti C, Ferrigno G and De Momi E. (2016) Gesteme-free context-aware adaptation of robot behaviour in human-robot cooperation, Artificial Intelligence in Medicine, 74, pp. 32-43.

57. De Momi E, Ferrigno G. (2010) Robotic and artificial intelligence for keyhole neurosurgery: The Robocast project, a multi-model autonomous path planner, Proc. Inst. Mech. Eng. H: J. Eng. Med. 244(5), 715 - 727.

\section{Appendix A}

Name:

Age: (in years)

Sex: (male/female)

Purpose: The purpose of this questionnaire is to evaluate your procedural skills and your understanding of different surgical contexts to accomplish Thoracentesis procedure.

\section{Skills predicted:}

A. Knowledge application: Use your knowledge to answer the questions on Thoracentesis procedure

B. Information interpretation: This skill represents your understanding for Thoracentesis procedure

Instructions: You will have to complete 12 questions. There may be none, one or more than one right answers. 


\section{Questions}

1) How many phases are required to complete to accomplish Thoracentesis procedure?
$\square 2 \quad \square 4 \quad \square 3 \quad \square 8 \quad \square 6 \quad \square 5$
If you want to specify any details,

2) How many phases require excellent technical skills, e.g. dexterity, to accomplish the Thoracentesis procedure?

\section{$\square 2 \quad \square 4 \quad \square 3 \quad \square 1 \quad \square 6 \quad \square 5$}

3) Which is/are the following instrument/instruments is required during the anaesthesia?
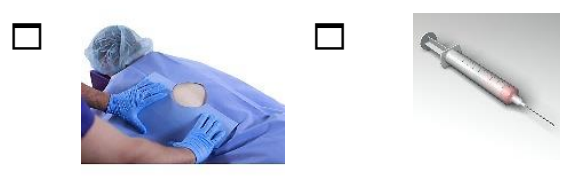

4) Is $50 \mathrm{~mL}$ syringe used after anaesthetising the skin?
True $\square$ False

5) Which of the following is true about Thoracentesis procedure?

The procedure is performed on chest cavity

$\square$ Three-way stopcock is used in anaesthesia phase

$\square$ Three-way stopcock is used on chest (location)

$50 \mathrm{~mL}$ syringe is used in aspiration phase

all above are true

If you want to specify any details,

6) When do you turn the lever of the stopcock to the direction of syringe?

After inserting needle in the chest

$\square$ For removing the fluid

$\square$ To insert the fluid

$\square$ To insert anaesthetic agent

If you want to specify any details,

7) How many instruments/material items are required to accomplish Thoracentesis?
$\square$

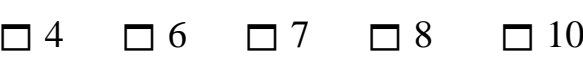


8) Which anatomical region do you require to anaesthetise during anaesthesia phase?

Skin of the chest

Area of insertion - intercostal space

Area of insertion - paravertebral space

Area of insertion - between eight and ninth rib

Area of insertion - skin

If you want to specify any details,

9) Which anatomical region do you need to penetrate to reach the thoracic cavity?

Skin of the chest

Area of insertion - intercostal space

Area of insertion - paravertebral space

Area of insertion - between eight and ninth rib

Area of insertion - skin

If you want to specify any details,

10) Which instruments/instrument do you use after using the syringe in anaesthesia and in penetration phase?

Betadine swab

$\square$ Flexible catheter

$\square 0 \mathrm{~mL}$ syringe

$\square$ Large needle

$\square$ Three-way stopcock

$\square$ Information not available

11) How many steps are required to complete Thoracentesis procedure?
$4 \square 6$
$\square 3$
$\square 1$
$\square 5$
$\square 8$

12) Which are of the following steps corresponding to anaesthesia and aspiration phase?

Anaesthetise $\square$ Mark $\square$ Turn lever to drainage bag $\square$ None of these $\square$ All 